\title{
Strain and Electronic Nematicity in $\mathrm{La}_{2-x} \mathrm{Sr}_{x} \mathrm{CuO}_{4}$
}

\author{
Anthony T. Bollinger ${ }^{1} \bullet$ Ze-Bin $\mathrm{Wu}^{1} \bullet$ Longlong $\mathrm{Wu}^{1} \bullet \mathrm{Xi} \mathrm{He}^{1,2} \bullet$ Ilya Drozdov ${ }^{1} \bullet$ Jie $\mathrm{Wu}^{1} \bullet \operatorname{lan}$ \\ Robinson $^{1} \bullet$ Ivan Božović ${ }^{1,2}$ \\ ${ }^{1}$ Condensed Matter Physics and Materials Science Department, Brookhaven National Laboratory, \\ Upton, NY 11973, USA \\ ${ }^{2}$ Applied Physics Department, Yale University, New Haven, CT 06520, USA
}

Keywords: Electronic nematicity, Spontaneous symmetry breaking, Strain, Cuprates, Hightemperature superconductivity, Transverse voltage, Electrical transport

\begin{abstract}
Electronic nematicity has previously been observed in $\mathrm{La}_{2-x} \mathrm{Sr}_{x} \mathrm{CuO}_{4}$ thin films by the angle resolved transverse resistivity method with a director whose orientation is always pinned to the crystal axes when the film is grown on an orthorhombic substrate but not when the substrate is tetragonal. Here we report on measurements of thin films grown on (tetragonal) $\mathrm{LaSrAlO}_{4}$ and subsequently placed in an apparatus that allows the application of uniaxial compressive strain. The apparatus applied enough force to produce a $1 \%$ orthorhombicity in $\mathrm{LaSrAlO}_{4}$ and yet no change in the electronic nematicity was observed in films under strain compared to when they were unstrained. The lattice effects are weak, and the origin of nematicity is primarily electronic.
\end{abstract}




\section{Introduction}

An intriguing new electronic state, the electronic nematic [1], has recently been revealed by a wide range of different techniques in various materials. This spontaneous breaking of the rotational symmetry of the electron liquid with respect to the crystal lattice has emerged as a seemingly ubiquitous characteristic of the normal state of many unconventional superconductors, having been observed in the cuprates [2-11], ruthenates [12,13], iron based [14-19], heavy fermion [20-22], and topological superconductors [23]. In one example, the high temperature superconductor $\mathrm{La}_{2-x} \mathrm{Sr}_{x} \mathrm{CuO}_{4}$, the resistivity has been shown to be anisotropic for underdoped single crystals [2] and subsequently across most of the phase diagram in epitaxial thin films [9-11]. The thin film data displays oscillations in the resistivity both parallel and perpendicular to the flow of electrical current in the absence of an applied magnetic field. These oscillations have a $\mathbf{C}_{2}$ symmetry rather than the $\mathbf{C}_{4}$ symmetry of the tetragonal films. They were most easily detected in films on the underdoped side of the phase diagram, with the oscillation amplitude decreasing exponentially with doping. The orientation of the director of nematicity, i.e. the angle of maximum longitudinal resistivity and zero transverse resistivity, did not coincide with the crystal axes, as was shown to occur in orthorhombic films. The director orientation was, however, reproducible with doping but varied with temperature for films with nominal doping close to optimal. Despite all that is known about the nematicity in cuprates its microscopic origin remains a puzzle. If the nematicity in a thin film could somehow be altered externally then the new information gained could perhaps shed light on this mystery. Here we report our efforts to alter the nematicity of $\mathrm{La}_{2-x} \mathrm{Sr}_{x} \mathrm{CuO}_{4}$, thin films by externally applied strain. 


\section{Measurements and Results}

We have used the angle resolved transverse resistivity method in this work as it is well suited for examining electronic nematicity in thin films [9-11]. The most straightforward way to apply this method is to pattern a thin film into several Hall bars, all oriented at different angles with respect to the film's principal crystal axes, and then measure the longitudinal and transverse resistivity in each. An example is the "sunbeam" type pattern shown in Fig. 1. A large central contact pad serves as a common drain (I-) for each Hall bar while current is supplied at an individually addressed source electrode (I+). The Hall bar structures constrain the current to flow, on average, in one direction. As shown in Fig. 1b, several voltage electrodes can be connected to each Hall bar with which to obtain statistics on the longitudinal and transverse resistivities at many different angles. Electronic nematicity produces measurable oscillations in both of these resistivities. However, the amplitude of the oscillation is typically quite small when compared to the average longitudinal resistivity and can be easily obscured by spurious extrinsic factors, such as device-to-device variations due to substrate imperfections, lithography, sample inhomogeneity, or variations in the film thickness. The angle-averaged transverse resistivity, on the other hand, is always zero (in the absence of a magnetic field) and consequently most easily reveals the oscillations due to nematicity [9].

There are a few conditions that must be fulfilled for this method to be applied successfully. First, there should be enough bars with different orientations that the entire range of angles is covered quite densely - a structure with only four or eight bars is typically not sufficient to determine the amplitude of nematicity and director orientation $[10,11]$. This effectively constrains the sample to be a lithographically patterned thin film. A thin slice of a 
single crystal could be carefully patterned by focused ion beam, in principle, but the time involved would be so high as to be prohibitive. Second, the sample must be very homogeneous, both in terms of stoichiometry and crystal structure. Since numerous Hall bars are involved in this type of measurement, compositional variations throughout the sample that influence its intrinsic resistivity can spoil the ability to resolve the relatively small angular resistivity oscillations due to nematicity. Variations in the orientation of the crystal structure are similarly detrimental; deposition methods that produce epitaxial films therefore are essential. The film thickness should be constant for all the devices.

To examine the effects of strain on the electronic nematicity in $\mathrm{La}_{2-x} \mathrm{Sr}_{x} \mathrm{CuO}_{4}$ we start with high-quality thin films grown by atomic-layer-by-layer molecular beam epitaxy. We pattern them into the sunbeam configuration shown in Fig. 1 by standard microfabrication techniques. Longitudinal and transverse resistivity are then determined for each Hall bar from the average of 200 current-biased delta-mode measurements of voltage across the four longitudinal and three transverse pairs of contacts. The samples were held at $300 \pm 0.1 \mathrm{~K}$ in $1 \mathrm{~atm}$ of nitrogen. The resulting transverse resistivity for an underdoped $\mathrm{La}_{1.98} \mathrm{Sr}_{0.02} \mathrm{CuO}_{4}$ thin film is shown in Fig. 2a. It shows a $180^{\circ}$ periodic oscillation, like in Ref. 9, and with a similar amplitude.

Strain was introduced into the patterned thin film by means of a homemade clamping device. The sample is placed inside this fixture, which contacts the $10 \mathrm{~mm} \times 10 \mathrm{~mm} \times 1 \mathrm{~mm}$ sample substrate on all four of its $10 \mathrm{~mm} \times 1 \mathrm{~mm}$ "side" faces and the $10 \mathrm{~mm} \times 10 \mathrm{~mm}$ "backside" face that has no film on it. The parts of the fixture that contact the backside and two of the side faces, all three mutually perpendicular, are fixed. The other two edge faces touch linearly movable pieces of the clamp. Two fine-pitch set screws, one for each side, push on these pieces. 
This generates large forces on the sides of the substrate and introduces strain in two perpendicular directions into the film.

In Fig. $2 b$ we show the transverse resistivity for one Hall bar of the $\mathrm{La}_{1.98} \mathrm{Sr}_{0.02} \mathrm{CuO}_{4}$ film under the influence of different levels of uniaxial strain, as quantified by the torque applied to the set screw (only one set screw was used, the other was loose). The transverse resistivity is essentially constant within the error bars for all levels of strain. Application of larger strain than the maximum shown in Fig. $2 \mathrm{~b}$ resulted in sudden simultaneous multiple fracturing events and complete loss of the sample. Results obtained using another film with nominally higher doping, $\mathrm{La}_{1.80} \mathrm{Sr}_{0.20} \mathrm{CuO}_{4}$, are shown in Fig. 3. The amplitude of transverse resistivity oscillations is two orders of magnitude lower due to the increased number of carriers, as expected [9]. Measurements taken in the unstrained and strained conditions essentially fall on top of one another, confirming that the amplitude of oscillations does not change with the strain. Another consequence from the data in Fig. 3 is that not only is the amplitude unaffected by the application of strain but the offset angle, $\alpha$, i.e., the orientation of the nematic director, does not change either.

X-ray diffraction (XRD) was subsequently used to quantify the amount of strain introduced into the film by the clamping fixture. The XRD measurement was performed using a Bruker D8

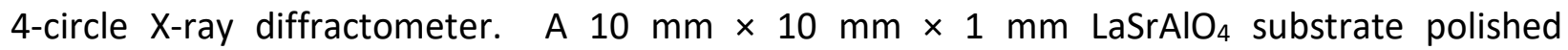
perpendicular to the crystallographic [001] direction was held by the clamping apparatus, which was then fixed onto the sample stage by vacuum. The walls of the fixture are higher than the substrate thickness so the substrate is recessed inside of the apparatus (Fig. 4a). As a trade-off then between the signal intensity and the accessibility of the X-ray beam, the incident and 
diffraction beams were initially set to be symmetric about the normal of the (118) crystal plane

(Fig. 4b). Then the sample was rotated and the lattice constants were determined based on the

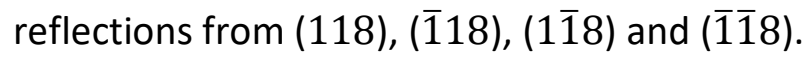

The measurement was first conducted with the substrate in a strain-free state to determine the intrinsic lateral lattice constants, which are $3.762 \AA$ and $3.765 \AA$, i.e. the $\mathrm{LaSrAlO}_{4}$ is essentially tetragonal. Then the measurement was conducted in the strained condition, where the substrate was pressed along one axis, while the other (perpendicular) in-plane axis of the substrate was set free for deformation. The torque on the screw was pre-loaded to $0.28 \mathrm{~N}-\mathrm{m}$, which generated an axial force of $\sim 500 \mathrm{~N}$ to the end of the substrate. The lattice constants changed to $3.746 \AA$ and $3.779 \AA$ as a result of this force, indicating that a $\sim 0.4 \%$ strain is present in the substrate and proving that the clamping fixture is indeed capable of generating large deformations of the crystal lattice.

The observation that the application of uniaxial strain converted the tetragonal substrate to an orthorhombic one while maintaining the same nematic director orientation throughout is unexpected, given what has been seen previously in $\mathrm{La}_{2-x} \mathrm{Sr}_{x} \mathrm{CuO}_{4}$ [9] and $\mathrm{Sr}_{2} \mathrm{RuO}_{4}$ [13] films. In prior work, it was shown that the director was not determined by the crystal axes of the substrate when the films were grown on tetragonal substrates. If the substrates were orthorhombic, on the other hand, the director would align with one of the crystal axes. In what follows, we discuss various possible explanations for this difference.

First, we note that in this work the XRD data were taken on a blank substrate while the transport measurements are made on a patterned film. Perhaps the strain applied in the two 
cases was not identical, even though the clamp conditions were the same. It seems quite unlikely, though, that the strain in the second case could have been negligibly small, given that the clamp can exert enough force that the samples used for transport were eventually crushed in the straining process. Still, in future experiments, we plan to directly measure the amount of strain present during transport by a modified sample arrangement where half the film is patterned into a semi-sunbeam for transport while the other half is left untouched for XRD, with both measurements performed simultaneously.

Second, compressing the samples in our clamping apparatus not only distorts the crystal lattice but the sunbeam pattern as well, adding some error to the determination of the director angle. However, the orthorhombicity induced by strain is $\sim 0.9 \%$ so the small error that this introduces in the distorted sunbeam pattern angle is less than $0.3^{\circ}$. This is at least two orders of magnitude smaller than the initial offset angle of $\alpha=54.7^{\circ}$ in the unstrained condition. Clearly the orientation of the director does not align with the crystal axes in the strained case, even though it appears to be orthorhombic.

Third, in this work the substrate becomes orthorhombic by an externally applied compressive strain, whereas in previous studies orthorhombic films were obtained by epitaxial strain due to films grown on orthorhombic $\mathrm{NdGaO}_{3}$ substrates polished perpendicular to the [110] crystal direction. The surface lattice constants of these $\mathrm{NdGaO}_{3}$ substrates are $\left(a^{2}+b^{2}\right)^{1 / 2}$ $=7.727 \AA$, and $c=7.708 \AA$. In principle, thin films of $\mathrm{La}_{2-x} \mathrm{Sr}_{x} \mathrm{CuO}_{4}$ grow with $2 \times 2$ unit cells in each of these spaces, resulting in lattice constants of $3.863 \AA$ and $3.854 \AA$, both of which are larger than the $\mathrm{La}_{2-x} \mathrm{Sr}_{x} \mathrm{CuO}_{4}$ bulk lattice constants. Therefore, $\mathrm{La}_{2-x} \mathrm{Sr}_{x} \mathrm{CuO}_{4}$ films grow on $\mathrm{NdGaO}_{3}$ under substantial tensile strain in both the $a$ and $b$ directions. Films grown on tetragonal $<001>$ 
$\mathrm{LaSrAlO}_{4}(a=3.754 \AA$ ) , on the other hand, are under compressive epitaxial strain. It is possible that tensile strain strongly affects the orientation of nematic director, even locking it with the crystal axes, whereas compressive strain is essentially not relevant.

Finally, yet another possibility is that the key difference is that in one case the films are grown under orthorhombic strain, while in the other strain is applied on an already grown tetragonal film. If the films in fact contain domains of both orthorhombic orientations it is conceivable that external perturbation may affect more the domain distribution while they are being formed (at high growth temperature $T \approx 650^{\circ} \mathrm{C}$, or upon cooling down), than when they are already fixed and the topological barrier for reorientation is already too high.

Overall, it is fair to say that it is still unclear how strain affects electronic nematicity in the cuprates, let alone what the origin of nematicity may be - expect that it seems to be primarily electronic. Further experimenting with the overall strain in cuprate films, i.e. the combination of the epitaxial strain and externally applied strain, could shed more light on this subject. Applying compressive strain to films grown on $<110>\mathrm{NdGaO}_{3}$ is already possible and designing a different kind of mechanical apparatus to apply tensile strain to films grown on $<001>\mathrm{LaSrALO}_{4}$ is certainly feasible.

\section{Conclusion}

Externally applied strain in a mechanical clamping apparatus was shown to transform the crystal structure of $\mathrm{LaSrAlO}_{4}$ from tetragonal to orthorhombic. Despite this physical change, no effect was observed on the nematicity of $\mathrm{La}_{2-\mathrm{x}} \mathrm{Sr}_{x} \mathrm{CuO}_{4}$ thin films grown on these types of 
substrates as determined by the angle resolved transverse resistivity method. This is consistent with nematicity being of primarily electronic origin, with very weak coupling to the crystal lattice. However, it seems that lattice deformation has somewhat stronger effect on films growing on orthorhombic substrates, than on films grown on tetragonal substrates and then uniaxially strained.

\section{Acknowledgements}

The research at Brookhaven National Laboratory was supported by the U.S. Department of Energy, Basic Energy Sciences, Materials Sciences and Engineering Division. X. H. was supported by the Gordon and Betty Moore Foundation's EPiQS Initiative through Grant GBMF4410.

\section{References}

1. Kivelson, S.A., Fradkin, E., Emery, V.J.: Electronic liquid-crystal phases of a doped Mott insulator. Nature 393, 550-553 (1998)

2. Ando, Y., Segawa, K., Komiya, S., Lavrov, A.N.: Electrical Resistivity Anisotropy from SelfOrganized One Dimensionality in High-Temperature Superconductors. Phys. Rev. Lett. 88, 137005 (2002)

3. Abdel-Jawad, M., Kennett, M.P., Balicas, L., Carrington, A., Mackenzie, A.P., McKenzie, R.H., Hussey, N.E.; Anisotropic scattering and anomalous normal-state transport in a hightemperature superconductor. Nat. Phys. 21, 821-825 (2006) 
4. Daou, R., Chang, J., LeBoeuf, D., Cyr-Choinière, O., Laliberté, F., Doiron-Leyraud, N., Ramshaw, B.J., Liang, R., Bonn, D.A., Hardy, W.N., Taillefer, L.: Broken rotational symmetry in the pseudogap phase of a high-Tc superconductor. Nature $463,519-522$ (2010)

5. Lawler, M.J., Fujita, K., Lee, J., Schmidt, A.R., Kohsaka, Y., Kim, C.K., Eisaki, H., Uchida, S., Davis, J.C., Sethna, J.P., Kim, E.-A.: Intra-unit-cell electronic nematicity of the high- $T_{\mathrm{c}}$ copper-oxide pseudogap states. Nature 466, 347-351 (2010)

6. Li, L., Alidoust, N. Tranquada, J.M., Gu, G.D., Ong, N.P.: Unusual Nernst Effect Suggesting Time-Reversal Violation in the Striped Cuprate Superconductor $\mathrm{La}_{2-x} \mathrm{Ba}_{x} \mathrm{CuO}_{4}$. Phys. Rev. Lett. 107, 277001 (2011)

7. Fujita, K., Kim, C.K., Lee, I., Lee, J., Hamidian, M.H., Firmo, I.A., Mukhopadhyay, S., Eisaki, H., Uchida, S., Lawler, M.J., Kim, E.-A., Davis, J.C.: Simultaneous Transitions in Cuprate Momentum-Space Topology and Electronic Symmetry Breaking. Science 344, 612-616 (2014)

8. Zhao, L., Belvin, C.A., Liang, R., Bonn, D.A., Hardy, W.N., Armitage, N.P., Hsieh, D.: A global inversion-symmetry-broken phase inside the pseudogap region of YBa2Cu3Oy. Nat. Phys. 13, 250-354 (2017)

9. Wu, J., Bollinger, A.T., He, X., Božović, I.: Spontaneous breaking of rotational symmetry in copper oxide superconductors. Nature. 547, 432-435 (2017)

10. Wu, J., Bollinger, A.T., He, X., Božović, I.: Detecting Electronic Nematicity by the AngleResolved Transverse Resistivity Measurements. J. Supercond. Nov. Magn. 32, 1623-1628 (2018) 
11. Wu, J., Bollinger, A.T., He, X., Gu, G.D., Miao, H., Dean, M.P.M., Robinson, I.K., Božović, I.: Angle-Resolved Transport Measurements Reveal Electronic Nematicity in Cuprate Superconductors. J. Supercond. Nov. Magn. (2019) https://doi.org/10.1007/s10948-01905222-5

12. Borzi, R.A., Grigera, S.A., Farrell, J., Prry, R.S., Lister, S.J.S., Lee, S.L., Tennant, D.A., Maeno, Y., Mackenzie, A.P.: Formatiion of a Nematic Fluid at High Fields in $\mathrm{Sr}_{3} \mathrm{Ru}_{2} \mathrm{O}_{7}$. Science 315, 214-217 (2007)

13. Wu, J., Nair, H.P., Bollinger, A.T., He, X., Robinson, I., Schreiber, N.J., Shen, K.M., Schlom, D.G., Božović, I.: Electronic nematicity in $\mathrm{Sr}_{2} \mathrm{RuO}_{4}$. Submitted (2019)

14. Fernandes, R.M., Chubukov, A.V., Schmalian, J.: What drives nematic order in iron-based superconductors? Nat. Phys. 10, 97-104 (2014)

15. Avci, S., Chmaissem, O., Allred, J.M., Rosenkranz, S., Eremin, I., Chubukov, A.V., Bugaris, D.E., Chung, D.Y., Kanatzidis, M.G., Castellan, J.-P., Schlueter, J.A., Claus, H., Khalyavin, D.D., Manuel, P., Daoud-Aladine, A., Osborn, R.: Magnetically driven suppression of nematic order in an iron-based superconductor. Nat. Commun. 5, 3845 (2014)

16. Watson, M.D., Kim, T.K., Haghighirad, A.A., Davies, N.R., McCollam, A., Narayanan, A., Blake, S.F., Chen, Y.L., Ghannadzadeh, S., Schofield, A.J., Hoesch, M., Meingast, C., Wolf, T., Coldea, A.I.: Emergence of the nematic electronic state in FeSe. Phys. Rev. Lett. 91, 155106 (2015)

17. Baek, S.-H., Efremov, D.V., Ok, J.M., Kim, J.S., van den Brink, J., Büchner, B.: Orbital-driven nematicity in FeSe. Nat. Mater. 14, 210 (2015) 
18. Hosoi, S., Matsuura, K., Ishida, K., Wang, H., Mizukami, Y., Watashige, T., Kasahara, S., Matsuda, Y., Shibauchi, T.: Nematic quantum critical point without magnetism in $\mathrm{FeSe}_{1-x} \mathrm{~S}_{x}$ superconductors. Proc. Natl. Acad. Sci. U.S.A. 113, 8139-8143 (2016)

19. Licciardello, S., Buhot, J., Lu, J., Ayres, J., Kasahara, S., Matsuda, Y., Shibauchi, T., Hussey, N.E.: Electrical resistivity across a nematic quantum critical point. Nature 567, 213-217 (2019)

20. Varma, C.M., Zhu, L.: Helicity order: hidden order parameter in $\mathrm{URu}_{2} \mathrm{Si}_{2}$. Phys. Rev. Lett. 96, 036405 (2006)

21. Okazaki, R., Shibauchi, T., Shi, H.J., Haga, Y., Matsuda, T.D., Yamamoto, E., Onuki, Y., Ikeda, H., Matsuda, Y.: Rotational Symmetry Breaking in the Hidden-Order Phase of $\mathrm{URu}_{2} \mathrm{Si}_{2}$. Science 331, 439-442 (2011)

22. Ronning, F., Helm, T., Shirer, K.R., Bachmann, M.D., Balicas, L., Chan, M.K., Ramshaw, B.J., McDonald, R.D., Balakirev, F.F., Jaime, M., Bauer, E.D., Moll, P.J.W.: Electronic in-plane

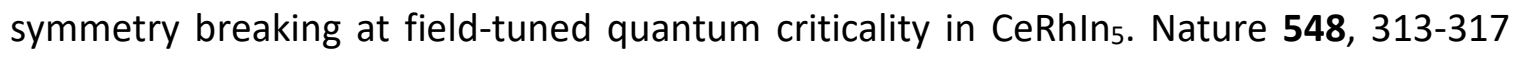
(2017)

23. Sun, Y., Kittaka, S., Sakakibara, T., Machida, K., Wang, J., Wen, J., Xing, X., Shi, Z., Tamegai, T.: Quasiparticle Evidence for the Nematic State above $\mathrm{Tc}_{\text {in }} \mathrm{Sr}_{x} \mathrm{Bi}_{2} \mathrm{Se}_{3}$. Phys. Rev. Lett. 123, 027002 (2019) 


\section{(a)}

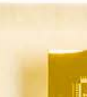

\section{国}

(b)

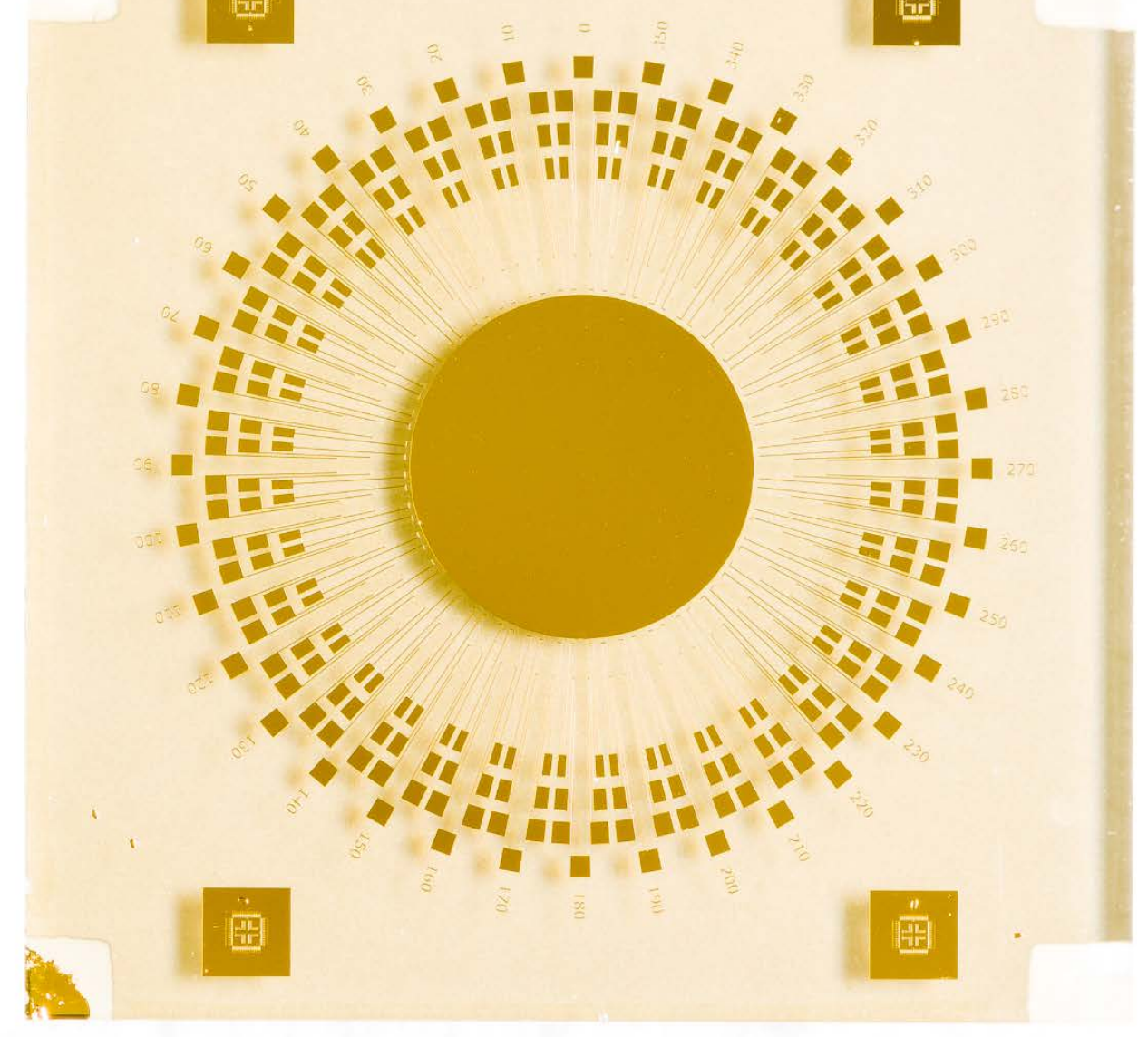

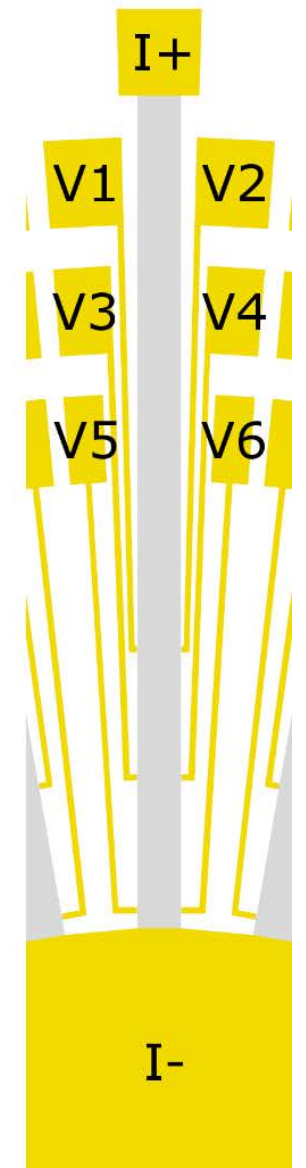

Figure 1. a Example of a sunbeam patterned sample used in the angle resolved transverse resistance measurements. b Diagram of one ray of the sunbeam pattern. Current is passed between the I+ and I- contacts. Longitudinal resistances are determined by measuring the voltage drops between pairs of contacts along each ray (V1-V3, V2-V4, V3-V5, and V4-V6) and transverse resistances from the voltage drops across each ray (V1-V2, V3-V4, V5-V6). 

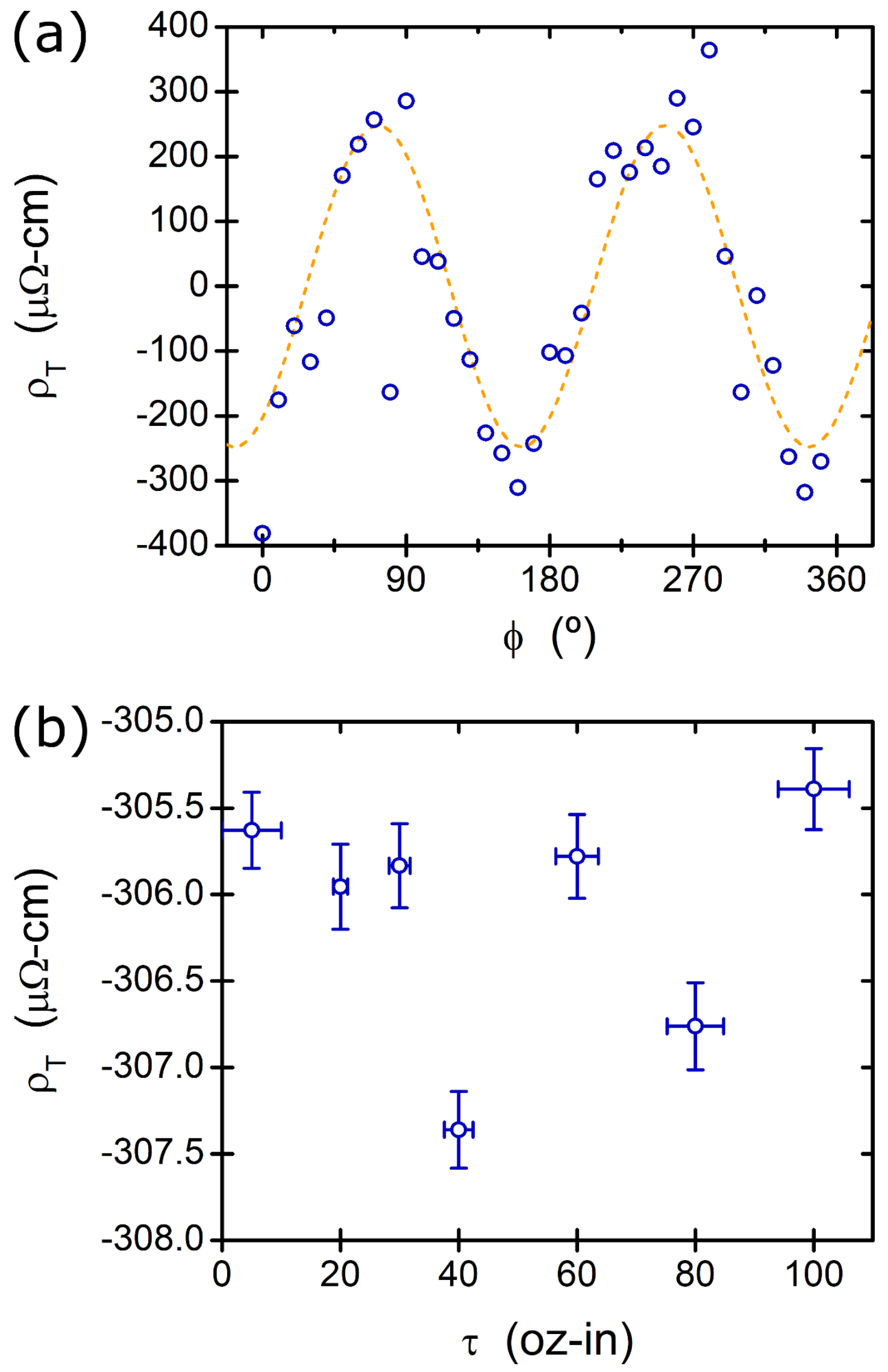
Figure 2. Transverse resistivity for a 20 unit cell thick $\mathrm{La}_{1.98} \mathrm{Sr}_{0.02} \mathrm{CuO}_{4}$ thin film grown on a $\mathrm{LaSrAlO}_{4}$ substrate. The Hall bars of each ray are $100 \mu \mathrm{m}$ wide and the spacing between voltage electrodes along the length of the bars is $300 \mu \mathrm{m}$. The data (o) are determined from the average of the three pairs of transverse voltage contacts on each ray. a The angular data taken without externally applied strain displays a $\mathbf{C}_{2}$ symmetry. The dashed line is a fit to the function $\rho_{\mathrm{T}}=\rho_{\mathrm{T}^{0}} \sin (2(\phi-\alpha))$, with $\rho_{\mathrm{T}^{0}}=247.8 \mu \Omega-\mathrm{cm}$ and $\alpha=27.4^{\circ}$. b The transverse resistivity as a function of torque on the jig screw is essentially constant. Here we show the data from the $160^{\circ}$ ray. 


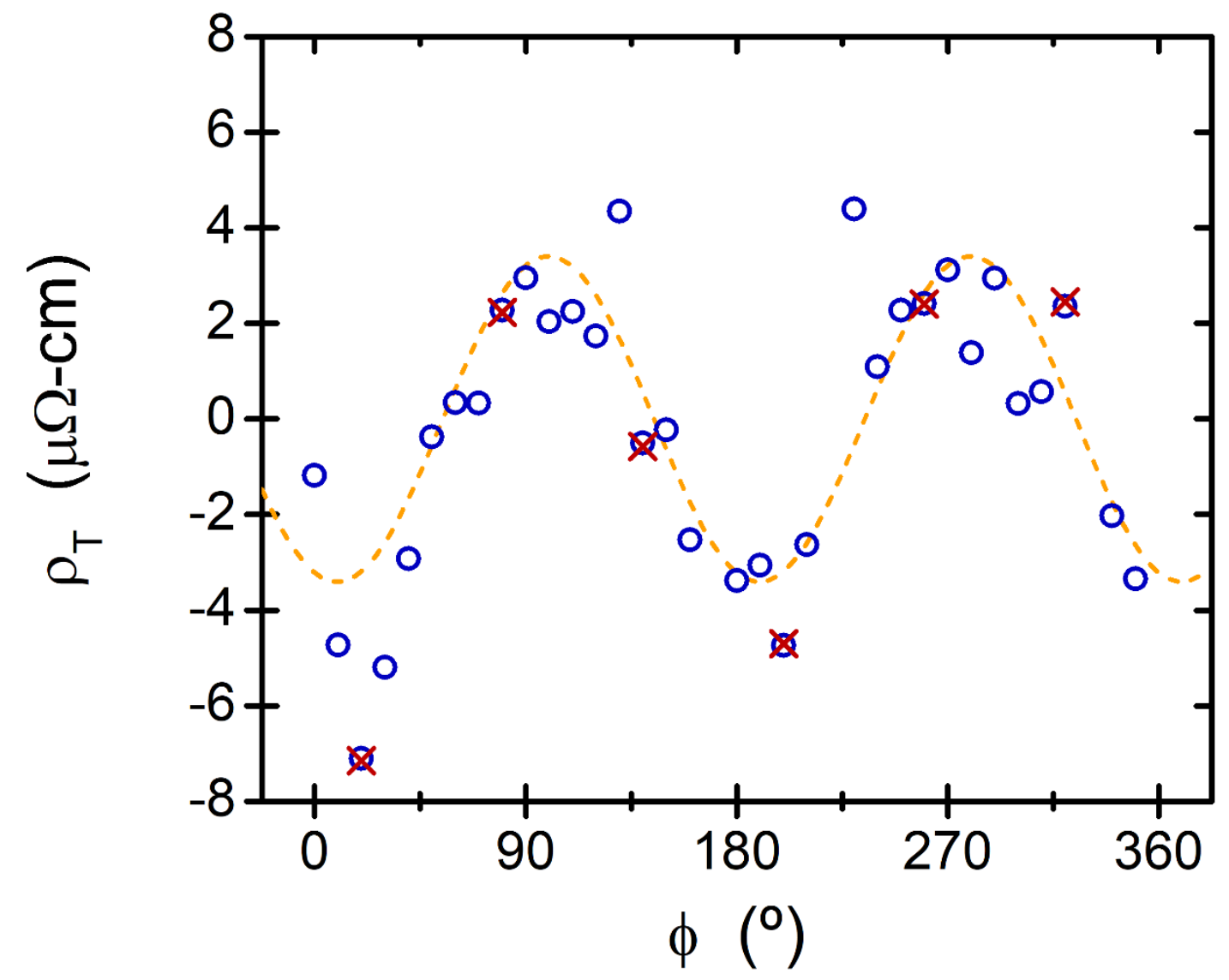

Figure 3. Transverse resistivity for a 20 unit-cell thick $\mathrm{La}_{1.80} \mathrm{Sr}_{0.20} \mathrm{CuO}_{4}$ film grown on a $\mathrm{LaSrAlO}_{4}$ substrate. The Hall bar dimensions are the same as in Fig. 2 and as in that case the data display a $C_{2}$ symmetry and the dashed line is a fit to the function $\rho_{\mathrm{T}}=\rho_{\mathrm{T}}{ }^{0} \sin (2(\phi-\alpha))$, with $\rho_{\mathrm{T}}{ }^{0}=3.4 \mu \Omega-\mathrm{cm}$ and $\alpha=54.7^{\circ}$. The data taken while the film is strained $(x)$ essentially fall right on top of the unstrained film data (o). The angle $\phi$ is the orientation of the Hall bar relative to the (100) direction without any externally applied strain. When the strain is applied, the pattern becomes slightly distorted, introducing an error less than $0.3^{\circ}$. The clamp set screw was torqued to 0.28 $\mathrm{N}-\mathrm{m}$ in the strained case. 

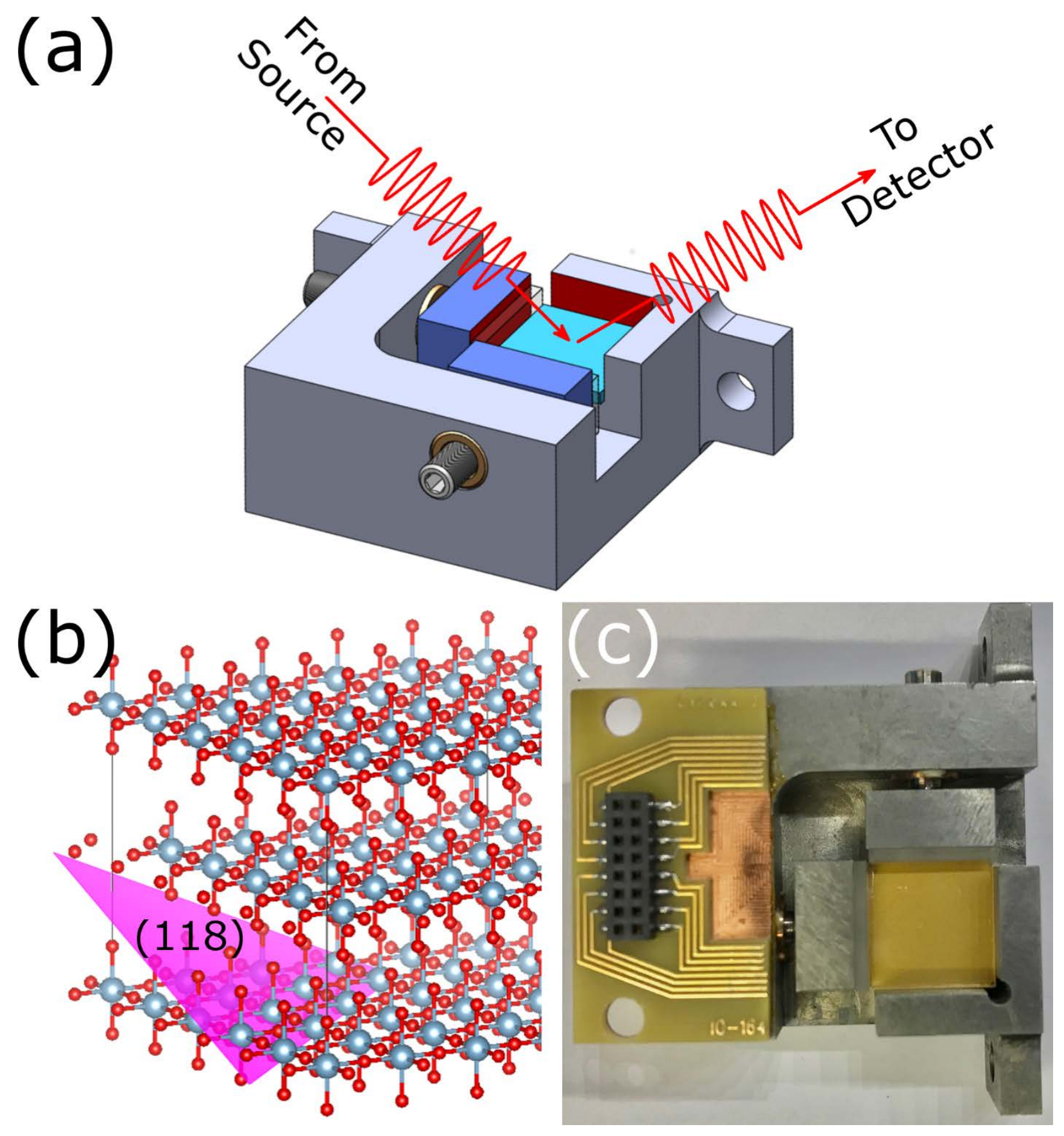

Figure 4. a Diagram of the $x$-ray diffraction measurement configuration. The sample is held by the clamping fixture and uniaxial strain is applied by turning one of the fine pitch screws with the aid of an adjustable torque screwdriver. b Since the sample is recessed in the clamp, the (118) planes were used for measurement. c Photograph of the apparatus used to apply strain. A blank 
substrate is in the sample position for demonstration purposes. The socket glued to the top of the fixture is for making electrical connections to patterned films by wire bonding and interfacing with the measurement electronics. 